\title{
Magnetic Moments of 70-Plet Baryons in Quark Model and QCD Sum Rules
}

\author{
T. M. Aliev ${ }^{1}$ and V.S. Zamiralov ${ }^{2}$ \\ ${ }^{1}$ Department of Physics, Middle East Technical University, 06800 Ankara, Turkey \\ ${ }^{2}$ Skobeltsyn Institute of Nuclear Physics, Lomonosov Moscow State University, Moscow 119991, Russia \\ Correspondence should be addressed to T. M. Aliev; taliev@metu.edu.tr
}

Received 21 July 2015; Accepted 27 September 2015

Academic Editor: Ming Liu

Copyright (C) 2015 T. M. Aliev and V. S. Zamiralov. This is an open access article distributed under the Creative Commons Attribution License, which permits unrestricted use, distribution, and reproduction in any medium, provided the original work is properly cited. The publication of this article was funded by SCOAP ${ }^{3}$.

Magnetic moments of the positive parity 70-plet baryons are estimated in the framework of the nonrelativistic quark model and QCD sum rules method. It is found that the magnetic moments of the 70-plet baryons can be expressed in terms of the $F$ and $D$ couplings and exhibit unitary symmetry. The QCD sum rules for the magnetic moments of the 70-plet octet baryons are formulated. A comparison of our results on magnetic moments of 56-plet and 70-plet baryons predicted from QCD sum rules is presented.

\section{Introduction}

Study of the electromagnetic properties of hadrons represents very important source of information about their internal structure and can provide valuable insight into understanding the mechanism of strong interactions at low energies, that is, about nonperturbative aspects of QCD. Particular interest deserves magnetic moments of baryons as a subject of permanent study due to growing experimental information [1].

Magnetic moments of the positive parity octet and decuplet baryons are studied in framework of different approaches, such as nonrelativistic quark model (NRQM) [1], static quark model [2], QCD string approach [3], chiral perturbation theory [4], Skyrme model [5], traditional QCD sum rules [6], light-cone version of QCD sum rules (LCSR) $[7,8]$, lattice QCD [9], SU(3) chiral quark soliton model [10], relativistic quark model with chiral symmetry [11], quenched chiral perturbation theory [12], and color-dielectric model [13].

Although the magnetic moments of the positive baryons are widely studied experimentally and theoretically, there is very limited information about their negative parity counterparts. Magnetic moments of these states can be extracted through the bremsstrahlung processes in photo and electroproduction reactions.

There exist few theoretical works, focusing on the magnetic moments of the negative parity baryons in framework of LCSR [14-16], effective Hamiltonian approach in QCD [17], constituent quark model [18], chiral quark model [19], unitarized chiral perturbation theory [20], chiral constituent quark model [21], and lattice QCD [22].

The positive octet and decuplet baryons usually enter the 56-plet and negative parity baryons lie in the 70-plet representation of the $\mathrm{SU}(6)$, which is the group-theoretical basis of the NRQM. In the unitary SU(3) model coupling constants of photons (and vector mesons) with baryons are expressed in terms of the $F$ and $D$ constants. In chiral model [19] mixing of 56-plet and 70-plet is studied for $1 / 2^{+}$and $3 / 2^{+}$ baryons.

The basic element of QCD sum rules calculation is the interpolating current of the corresponding hadron which can be reduced to 56-plet wave function in nonrelativistic limit. Here we note that in studying the properties of the $1 / 2^{-}$baryons the interpolating current for the positive parity baryons has been used although it has no usual NRQM limit for the 70-plet baryons. Despite this difficulty we will try to 
write QCD sum rules for such a way as to respecting the NRQM limit.

\section{2. $F$ and $D$ Couplings in Quark-Diquark Model}

Although the NRQM results of magnetic moments cannot be expressed in terms of $F$ and $D$ couplings, in [23], it was shown that they can be achieved in framework of diquarkquark model. The characteristic property of this model is that the photon or vector boson fields are to interact with diquark and single quark in a different way.

Let us discuss the magnetic moments of 56-plet baryons in NRQM in framework of diquark-quark model. As an example let us consider a proton from 56-plet. Its wave function can be written as

$$
\begin{aligned}
& \sqrt{18}\left|p, s_{z}=\frac{1}{2}\right\rangle=\mid 2 u_{1} u_{1} d_{2}-u_{1} d_{1} u_{2}-d_{1} u_{1} u_{2} \\
& +2 u_{1} d_{2} u_{1}-u_{1} u_{2} d_{1}+2 d_{2} u_{1} u_{1}-u_{2} u_{1} d_{1} \\
& \left.\quad-u_{2} d_{1} u_{1}\right\rangle
\end{aligned}
$$

where subindices 1 and 2 mean quark spin-up and spindown, respectively. The wave functions of other members of octet baryons can be obtained from proton wave function by appropriate replacements of quark fields.

The results of the magnetic moments of the 56-plet baryons in terms of $F$ and $D$ couplings can be obtained by using their wave functions and introducing the following four different matrix elements:

$$
\begin{aligned}
& \left\langle q_{1} q_{1}, q_{2}^{\prime}\left|\widehat{w}_{q}\right| q_{1} q_{1}, q_{2}^{\prime}\right\rangle=w_{11}^{q}, \\
& \left\langle q_{1} q_{2}, q_{1}^{\prime}\left|\widehat{w}_{q}\right| q_{1} q_{2}, q_{1}^{\prime}\right\rangle=w_{12}^{q}, \\
& \left\langle q_{1} q_{1}, q_{2}^{\prime}\left|\widehat{w}_{q}^{\prime}\right| q_{1} q_{1}, q_{2}^{\prime}\right\rangle=v_{11}^{q}, \\
& \left\langle q_{1} q_{2}, q_{1}^{\prime}\left|\widehat{w}_{q}^{\prime}\right| q_{1} q_{2}, q_{1}^{\prime}\right\rangle=v_{12}^{q} .
\end{aligned}
$$

Using these matrix elements with $e_{q} \widehat{w}_{q} \sigma_{z}^{q}$ as the modified magnetic moment operator, the magnetic moments of $\Sigma^{0}$ and $\Lambda$ baryons in [23] are obtained:

$$
\begin{aligned}
\mu\left(\Sigma^{0}\right) & =\left(e_{u}+e_{d}\right) \frac{2}{3} w_{11}+\frac{1}{3} e_{s}\left(2 v_{11}-v_{12}\right) \\
& =\left(e_{u}+e_{d}\right) F+e_{s}(F-D), \\
\mu(\Lambda) & =\left(e_{u}+e_{d}\right)\left(F-\frac{2}{3} D\right)+e_{s}\left(F+\frac{1}{3} D\right),
\end{aligned}
$$

where $(2 / 3) w_{11}=F, w_{12}=D$, and $\left(2 v_{11}-v_{12}\right)=3(F-D)$ and also $v_{11}=D$ and $v_{12}=3 F-D$. These results show that the nonrelativistic diquark-quark model reveals the unitary symmetry pattern and the magnetic moments of baryons can be formulated in terms of the $F$ and $D$ couplings [23, 24].
If we put $e_{u}=2 / 3$ and $e_{d}=e_{s}=-1 / 3$, from (3), one can easily obtain well known $\mathrm{SU}(3)$ relations between magnetic moments of $(1 / 2)^{+}$baryons [25]:

$$
\begin{aligned}
& \mu(p)=\mu\left(\Sigma^{+}\right)=F+\frac{1}{3} D, \\
& \mu\left(\Sigma^{-}\right)=\mu\left(\Xi^{-}\right)=-F+\frac{1}{3} D, \\
& \mu(n)=\mu\left(\Xi^{0}\right)=-\frac{2}{3} D, \\
& \mu(\Lambda)=-\frac{1}{3} D .
\end{aligned}
$$

The NRQM results can be found if we take $F=(2 / 3) D$, $D=1$, and replace the electric charge of quarks by their magnetic moments; that is, $e_{q} \rightarrow \mu_{q}$.

Now let us analyze magnetic moment of baryons entering the 70-plet representation SU(6) in framework of NRQM. The 70-plet in NRQM has the following decomposition $70=$ $(8,2)+(10,2)+(8,4)+(1,2)$. The wave function of 70 -plet within the NRQM is obtained in numerous works (see [21] and references therein). Following [21], the wave function of $N^{*+}$ state in 70-plet with positive parity can be written as

$$
\begin{aligned}
& \sqrt{18}\left|N^{*+}\right\rangle=\mid 2 u_{1} u_{1} d_{2}-u_{1} d_{1} u_{2}-d_{1} u_{1} u_{2}+2 d_{1} u_{2} u_{1} \\
& -u_{1} u_{2} d_{1}-u_{1} d_{2} u_{1}+2 u_{2} d_{1} u_{1}-u_{2} u_{1} d_{1} \\
& \left.-d_{2} u_{1} u_{1}\right\rangle .
\end{aligned}
$$

Using this wave function, within the diquark-quark model for the magnetic moment of $N^{*+}$ we get

$$
\mu_{N^{*+}}=e_{u} \frac{2}{3} w_{12}+e_{d} \frac{1}{3}\left(2 v_{12}-v_{11}\right)
$$

Using definitions $w_{11}, w_{12}, v_{11}, v_{12}$ (see redefinitions after (3)) we have for magnetic moment of $N^{*+}$

$$
\mu_{N^{*+}}=e_{u} F+e_{d}(2 F-D) \text {. }
$$

Performing similar analysis for the magnetic moments of $\Sigma^{0 *}$ and $\Lambda^{*}$ baryons we obtain

$$
\begin{aligned}
& \mu_{\Sigma^{* 0}}=\frac{1}{2}\left(e_{u}+e_{d}\right) F+e_{s}(2 F-D), \\
& \mu_{\Lambda^{* 0}}=\left(e_{u}+e_{d}\right) \frac{1}{6}(9 F-4 D)+e_{s} \frac{1}{3} D .
\end{aligned}
$$

Transition moment $\mu_{\Sigma^{0 *}} \Lambda^{* 0}$ is obtained immediately from group-theoretical relation $[23,24]$

$$
\left\langle\Sigma_{d \leftrightarrow s}|O| \Sigma_{d \leftrightarrow s}\right\rangle-\left\langle\Sigma_{u \leftrightarrow s}|O| \Sigma_{u \leftrightarrow s}\right\rangle=\sqrt{3}\left\langle\Sigma^{0}|O| \Lambda\right\rangle,
$$

where $O$ is appropriate operator and reads

$$
\mu_{\Sigma^{* 0} \Lambda^{* 0}}=-\frac{3}{2}\left(e_{u}-e_{d}\right)\left(F-\frac{2}{3} D\right)
$$

and in the NRQM yields zero. 
Magnetic moments of other members of octet baryons can be found with the help of appropriate replacements of quark fields. In the limit $F=(2 / 3) D, D=1$ and replace $e_{q} \rightarrow \mu_{q}$; we get the NRQM result of the magnetic moments of 70-plet baryons. When, in (7), (8) take the relevant quark charges we get unitary symmetry results for the octet baryons in the 70-plet, similar to the relations for the 56-plet baryons (see (4)):

$$
\begin{aligned}
\mu\left(N^{*+}\right) & =\mu\left(\Sigma^{*+}\right)=\frac{1}{3} D \\
\mu\left(N^{* 0}\right) & =\mu\left(\Xi^{* 0}\right)=F-\frac{2}{3} D, \\
\mu\left(\Sigma^{*-}\right) & =\mu\left(\Xi^{*-}\right)=-F+\frac{1}{3} D \\
\mu\left(\Sigma^{* 0}\right) & =-\mu\left(\Lambda^{*}\right)=-\frac{1}{2}\left(F-\frac{2}{3} D\right), \\
\mu\left(\Sigma^{*} \Lambda^{*}\right) & =-\frac{3}{2}\left(F-\frac{2}{3} D\right) .
\end{aligned}
$$

Finally, magnetic moments of 70-plet octet baryons can be obtained from results of 56-plet baryons with the help of simple replacements by comparing (3), (7), and (8). For example, the magnetic moment of $\Sigma^{0}$ in 70 -plet can be achieved from 56-plet one by replacing coefficients of $F$ and $D$ terms:

$$
\begin{aligned}
& \text { for } \Sigma^{0} \text { baryons, } \\
& \qquad \begin{array}{l}
F:\left(e_{u}+e_{d}\right) \longrightarrow \frac{1}{2}\left(e_{u}+e_{d}+2 e_{s}\right), \\
(F-D): e_{s} \longrightarrow e_{s} .
\end{array}
\end{aligned}
$$

These relations constitute one of the main results in the present work.

\section{QCD Sum Rules for Magnetic Moments of 56-Plet and 70-Plet Baryons}

These results of the magnetic moments of octet baryons can also be obtained from the QCD sum rules method which exhibits unitary symmetry patterns of a sense that they can be represented in terms of only two independent $F$ and $D$ type functions. Note that the conclusion is true not only for photon but for any vector field too (see, e.g., [26]). The key object of the QCD sum rules method is the interpolating currents. In the nonrelativistic limit the interpolating current of baryons can be reduced to their wave functions [27].

The magnetic moments of the octet baryons in framework of traditional and light-cone versions of QCD sum rules are calculated in various works (see, e.g., $[6,8]$ and references therein).

The QCD sum rules method is based on the correlation function:

$$
\Pi=i \int d^{4} x e^{i p x}\left\langle 0\left|T\left\{\eta_{B}(x) \bar{\eta}_{B}(0)\right\}\right| 0\right\rangle_{\gamma},
$$

where $T$ is the time ordering operator, $\gamma$ means external electromagnetic field, and $\eta_{B}$ is the interpolating current carrying the same quantum numbers as the corresponding baryon $B$. As an example we present the interpolating current for $\Sigma^{0}$ baryon:

$$
\begin{aligned}
\eta_{\Sigma^{0}} & =\sqrt{\frac{1}{2}} \epsilon^{a b c}\left\{\left(u^{a T} C s^{b}\right) \gamma_{5} d^{c}-\left(s^{a T} C d^{b}\right) \gamma_{5} u^{c}\right. \\
& \left.+\beta\left(u^{a T} C \gamma_{5} s^{b}\right) d^{c}-\beta\left(s^{a T} C \gamma_{5} d^{b}\right) u^{c}\right\},
\end{aligned}
$$

where $a, b, c$ are color indices, $C$ is the charge conjugation operator, and $\beta$ is the arbitrary parameter $(\beta=-1$ corresponds to the so called Ioffe current).

In order to construct QCD sum rules for magnetic moments of the octet baryons the correlation function is calculated in terms of hadrons and quark-gluon degrees of freedom. By matching these two representations the QCD sum rules for the octet baryons magnetic moments are obtained [7]. The magnetic moments of octet baryons in general form can be written as

$$
\begin{aligned}
\lambda_{B}^{2} \mu_{B} e^{-m_{B}^{2} / M^{2}}= & e_{u} \Pi_{1}^{B}\left(u, d, s, M^{2}\right) \\
& +e_{d} \Pi_{1}^{B}\left(d, u, s, M^{2}\right) \\
& +e_{s} \Pi_{2}\left(u, d, s, M^{2}\right),
\end{aligned}
$$

where $\lambda_{B}$ and $\mu_{B}$ are the residue and magnetic moment of corresponding baryon, respectively, and $\Pi_{i}$ are the invariant functions in the coefficient of $\not p \notin q$ Lorentz structure and their expressions can be found in $[6,8]$. Using the SU(2) symmetry (15) can be written as

$$
\begin{aligned}
\lambda_{B}^{2} \mu_{B} e^{-m_{B}^{2} / M^{2}}= & \left(e_{u}+e_{d}\right) \Pi_{1}^{B}\left(u, d, s, M^{2}\right) \\
& +e_{s} \Pi_{2}^{B}\left(u, d, s, M^{2}\right) .
\end{aligned}
$$

Using the relation obtained in [28] which connected $\Lambda$ and $\Sigma^{0}$ interpolating currents one can easily find the expression for the magnetic moment of $\Lambda$-hyperon:

$$
\begin{aligned}
3 \mu(\Lambda)= & 2 \mu\left(\Sigma^{0}(d \longleftrightarrow s)\right)+2 \mu\left(\Sigma^{0}(u \longleftrightarrow s)\right) \\
& -\mu\left(\Sigma^{0}\right) .
\end{aligned}
$$

We can now predict the magnetic moments of the octet in 70-plet with positive parity if we assume that the transformations obtained in NRQM (see (3), (8), and (12)) hold in the case of QCD sum rules, that is, at the level of correlation functions $\Pi$ 's.

In this case even the explicit expressions of interpolating currents of octet baryons belonging to the 70-plet representation are not known; one can predict the magnetic moments of these baryons. 
TABle 1: Magnetic moments of 56-plet and 70-plet baryons.

\begin{tabular}{lcc}
\hline$\mu\left(B^{*}\right)$ & QCD SR $1 / 2^{+}[8]$ & This work $\left(1 / 2^{+}, 70\right.$-plet $)$ \\
\hline$\mu\left(N^{*+}\right)$ & 2.72 & 0.83 \\
$\mu\left(N^{* 0}\right)$ & -1.65 & 0.22 \\
$\mu\left(\Sigma^{*+}\right)$ & 2.52 & 0.70 \\
$\mu\left(\Sigma^{-}\right)$ & -1.13 & -1.13 \\
$\mu\left(\Sigma^{0}\right)$ & 0.70 & 0.11 \\
$\mu(\Lambda)$ & -0.50 & -0.11 \\
$\mu\left(\Xi^{0}\right)$ & -0.89 & 0.69 \\
$\mu\left(\Xi^{-}\right)$ & -1.18 & -1.18 \\
\hline
\end{tabular}

Using (8) and (12) as well as (16) and (17) for the magnetic moments of $\Sigma$ and $\Lambda$ baryons in 70-plet we get the following sum rules:

$$
\begin{aligned}
\lambda_{\Sigma^{*}}^{2} \mu_{\Sigma^{*}}^{2} e^{-m_{\Sigma^{*}}^{2} / M^{2}}= & \frac{1}{2}\left[e_{u}+e_{d}+2 e_{s}\right] \Pi_{1}^{B}+e_{s} \Pi_{2}^{B}, \\
\lambda_{\Lambda^{*}}^{2} \mu_{\Lambda^{*}}^{2} e^{-m_{\Lambda^{*}}^{2} / M^{2}}= & {\left[\frac{3}{2}\left(e_{u}+e_{d}\right)\right] \Pi_{1}^{B} } \\
& +\left[2\left(e_{u}+e_{d}\right)-e_{s}\right] \Pi_{2}^{B} .
\end{aligned}
$$

Comparing these equations with the sum rules for the $\Sigma$ and $\Lambda$ baryons from 56-plet we see that they have changed drastically.

In order to get the idea about the magnitude of the magnetic moments of 70-plet baryons, in the obtained sum rules, we put their experimentally measured mass and for residues we put their values for 56-plet baryons. Under this assumption, the magnetic moments of the positive parity 70plet baryons are calculated and their values are presented in Table 1 (the 2nd column). For completeness we also present the magnetic moments of 56-plet baryons in this table (the 1st column).

\section{Conclusion}

It is shown that octet baryons in the 70-plet can be analyzed in the way similar to those of 56-plet. In particular, magnetic moments are written in terms of the $F$ and $D$ quantities characteristics for octet coupling. Moreover the main formulas for the magnetic moments are written in such a way as to obtain the NRQM results as well as unitary symmetry ones. The QCD sum rules for the magnetic moment of 70-plet octet baryons are constructed. A comparison of the magnetic moments for $(1 / 2)^{+} 56$-plet and 70-plet baryons predicted from $\mathrm{QCD}$ sum rules is presented.

\section{Conflict of Interests}

The authors declare that there is no conflict of interests regarding the publication of this paper.

\section{References}

[1] K. A. Olive, K. Agashe, C. Amsler et al., "Review of particle physics ," Chinese Physics C, vol. 38, no. 9, Article ID 090001, 2014.

[2] J. Franklin, "Phenomenological quark model for baryon magnetic moments and beta decay ratios," Physical Review D, vol. 66, no. 3, Article ID 033010, 2002.

[3] B. O. Kerbikov and Y. A. Simonov, "Baryon magnetic moments in the QCD string approach," Physical Review D, vol. 62, no. 9, Article ID 093016, 2000.

[4] S. J. Puglia and M. J. Ramsey-Musolf, "Baryon octet magnetic moments in chiral perturbation theory: more on the importance of the decuplet," Physical Review D, vol. 62, no. 3, Article ID 034010, 2000.

[5] N. W. Park and H. Weigel, "Static properties of baryons from an SU(3) pseudoscalar vector meson lagrangian," Nuclear Physics A, vol. 541, no. 3, pp. 453-491, 1992.

[6] C. B. Chiu, J. Pasupathy, and S. J. Wilson, "Determination of baryon magnetic moments from QCD sum rules," Physical Review D, vol. 33, no. 7, pp. 1961-1973, 1986.

[7] T. M. Aliev, A. Ozpineci, and M. Savci, "Magnetic moments of decuplet baryons in light cone QCD," Physical Review D, vol. 62, Article ID 053012, 2000.

[8] T. M. Aliev, A. Özpineci, and M. Savc1, "Octet baryon magnetic moments in light cone QCD sum rules," Physical Review D, vol. 66, no. 1, Article ID 016002, 2002.

[9] D. B. Leinweber, T. Draper, and R. M. Woloshyn, "Decuplet baryon structure from lattice QCD," Physical Review D, vol. 46, no. 7, pp. 3067-3085, 1992.

[10] M. Wakamatsu and N. Kaya, "Magnetic moments of octet and decuplet baryons in the $S U(3)$ chiral quark soliton model," Progress of Theoretical Physics, vol. 95, no. 4, pp. 767-778, 1996.

[11] S. N. Jena and S. Panda, "Magnetic moments of octet baryons in a relativistic quark model with chiral symmetry," International Journal of Modern Physics A, vol. 8, no. 25, article 4563, 1993.

[12] M. J. Savage, "The magnetic moments of the octet baryons in quenched chiral perturbation theory," Nuclear Physics A, vol. 700, no. 1-2, pp. 359-376, 2002.

[13] M. Bae and J. A. McGovern, "Magnetic moments of the octet baryons in the colour-dielectric model," Journal of Physics G: Nuclear and Particle Physics, vol. 22, no. 2, pp. 199-214, 1996.

[14] T. M. Aliev and M. Savc1, "Magnetic moments of negative parity baryons in QCD," Physical Review D, vol. 89, no. 5, Article ID 053003, 2014.

[15] T. Aliev and M. Savci, "Magnetic moments of $J^{P}=2 / 3^{-}$baryons in QCD," Physical Review D, vol. 90, no. 11, Article ID 116006, 2014.

[16] T. M. Aliev, K. Azizi, T. Barakat, and M. Savc1, "Diagonal and transition magnetic moments of negative parity heavy baryons in QCD sum rules," Physical Review D, vol. 92, no. 3, Article ID 036004, 2015.

[17] I. M. Narodetskii and M. A. Trusov, "Magnetic moments of negative parity baryons from the effective Hamiltonian approach to QCD," JETP Letters, vol. 99, no. 2, pp. 57-62, 2014.

[18] W. T. Chiang, S. N. Yang, M. Vanderhaeghen, and D. Drechsel, "Magnetic dipole moment of the $S_{11}(1535)$ from the $\gamma p \rightarrow \gamma \eta p$ reaction," Nuclear Physics A, vol. 723, no. 1-2, pp. 205-225, 2003.

[19] H. Dahiya and M. Gupta, "Octet and decuplet baryon magnetic moments in the chiral quark model," Physical Review D, vol. 67, no. 11, Article ID 114015, 2003. 
[20] T. Hyodo, S. I. Nam, D. Jido, and A. Hosaka, "Magnetic moments of the $\mathrm{N}(1535)$ resonance in the chiral unitarymodel," http://arxiv.org/abs/nucl-th/0305023.

[21] N. Sharma, A. Martínez Torres, K. P. Khemchandani, and H. Dahiya, "Magnetic moments of the low-lying $1 / 2^{-}$octet baryon resonances," The European Physical Journal A, vol. 49, article 11, 2013.

[22] F. X. Lee and A. Alexandru, "Magnetic moments of negativeparity baryons from lattice QCD," in Proceedings of the 28th International Symposium on Lattice Field Theory, p. 148, Villasimius, Italy, June 2010.

[23] L. Gelmi, V. S. Zamiralov, and S. L. Lepshokov, "Quark-biquark structure of baryons: magnetic moments on QCD and in unitary symmetry," Moscow University Bulletin Series 3: Physics and Astronomy, vol. 2, p. 33, 1985.

[24] E. N. Bukina, V. M. Dubovik, and V. S. Zamiralov, "Gottfried's integral and valence quark contribution," Moscow University Bulletin Series 3: Physics and Astronomy, vol. 2, p. 5, 2000.

[25] S. Coleman and S. L. Glashow, "Electrodynamic properties of baryons in the unitary symmetry scheme," Physical Review Letters, vol. 6, no. 8, pp. 423-425, 1961.

[26] T. M. Aliev, A. Özpineci, M. Savc1, and V. S. Zamiralov, "Vector meson-baryon strong coupling contants in light cone QCD sum rules," Physical Review D, vol. 80, no. 1, Article ID 016010, 16 pages, 2009.

[27] B. L. Ioffe, "On the choice of quark currents in the QCD sum rules for baryon masses," Zeitschrift für Physik C: Particles and Fields, vol. 18, no. 1, pp. 67-68, 1983.

[28] A. Özpineci, S. B. Yakovlev, and V. S. Zamiralov, "QCD sum rules: intercrossed relations for the $\Sigma^{0}-\Lambda$ mass splitting," Modern Physics Letters A, vol. 20, no. 4, pp. 243-249, 2005. 

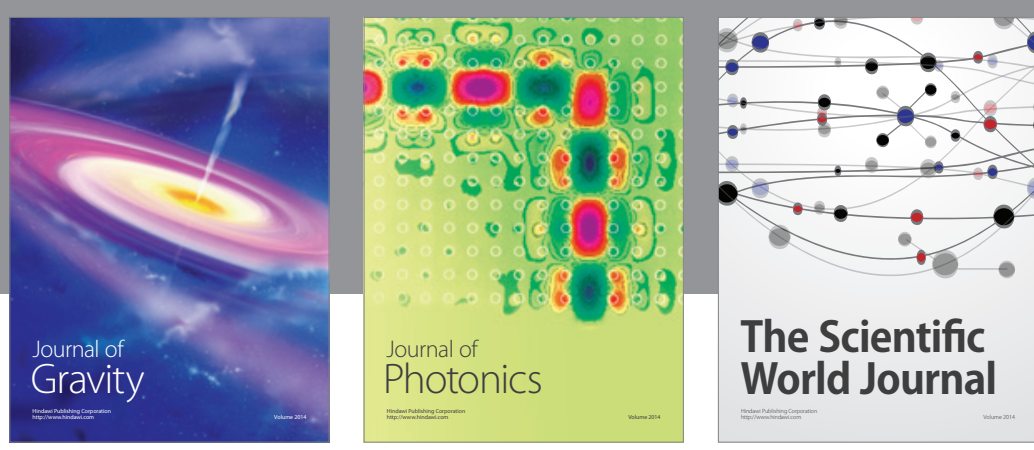

The Scientific World Journal
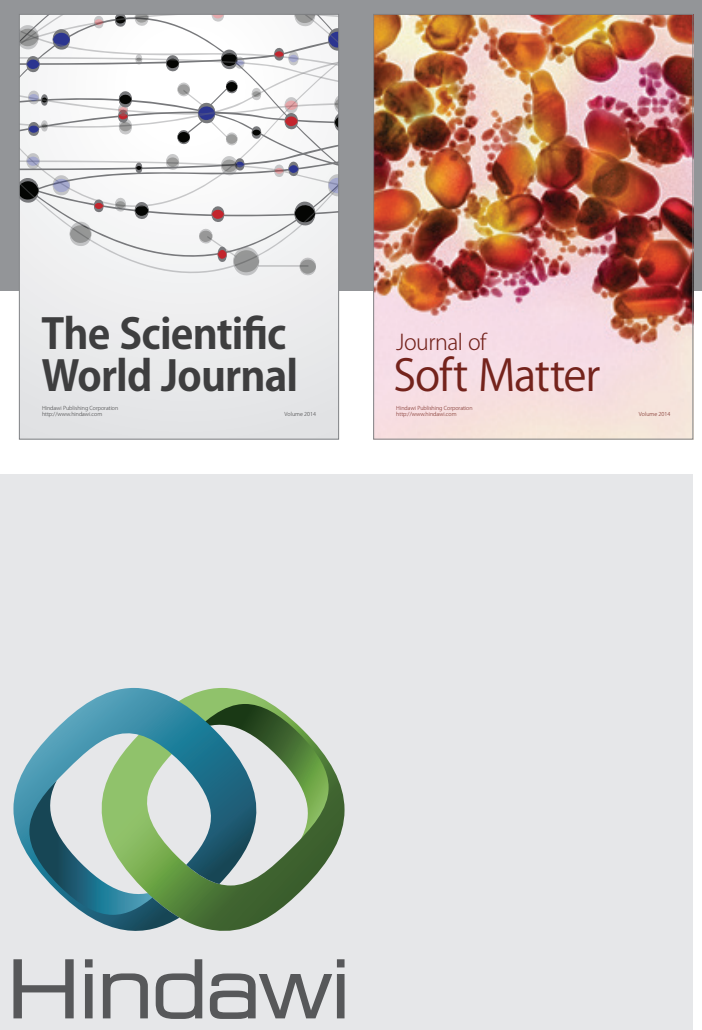

Submit your manuscripts at

http://www.hindawi.com

nternational Journal of

Statistical Mechanics
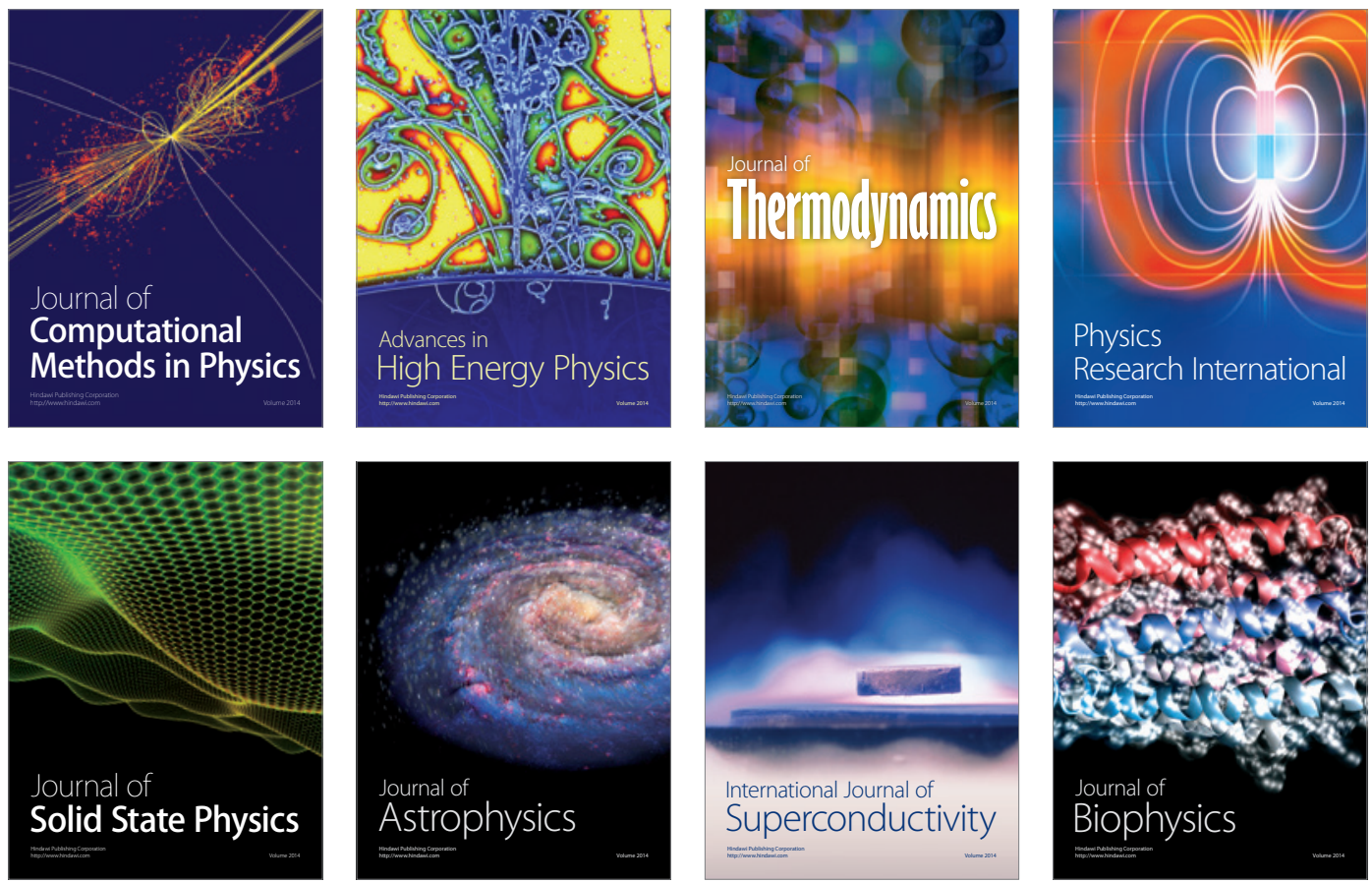
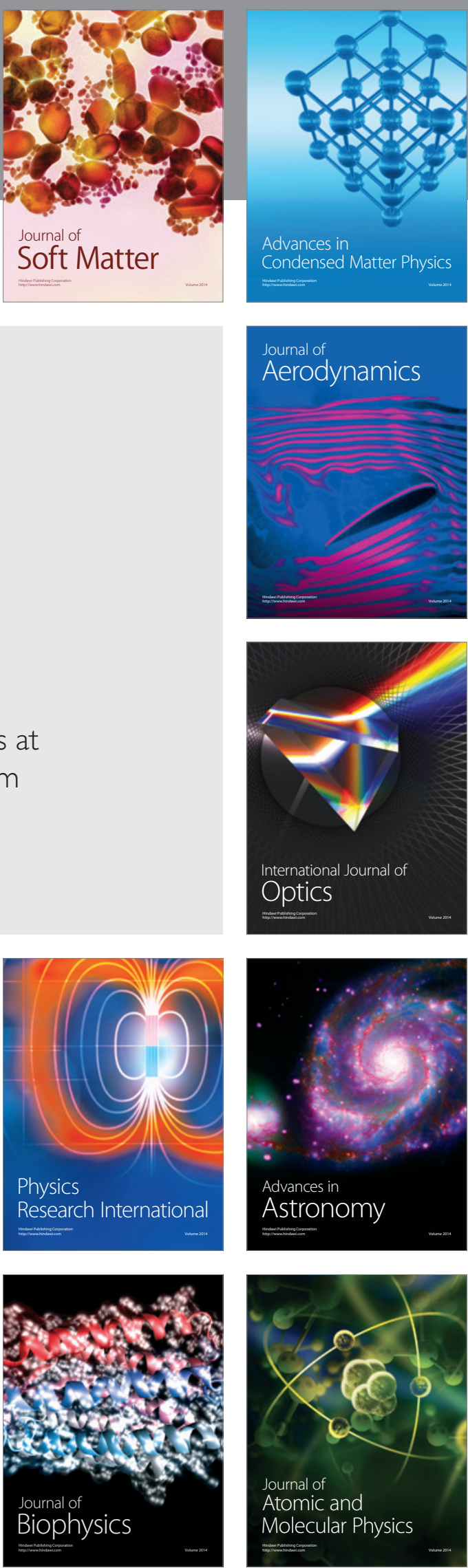